\title{
NUMERICAL INVESTIGATION ON FLUID FLOW OF PROTRUDING BRANCHING DUCT IN A DIVIDING JUNCTION OF A MINICHANNEL HEAT EXCHANGER
}

\author{
M. SETTATI, C. OLIET AND A. OLIVA \\ Heat and Mass Transfer Technological Center (CTTC), Universitat Politècnica de \\ Catalunya-BarcelonaTech (UPC) \\ ESEIAAT, Colom 11, 08222 Terrassa, Barcelona, Spain. \\ cttc@cttc.upc.edu, http://www.cttc.upc.edu
}

Key words: Protruding branch, Dividing junction, Distribution manifold, Minichannel heat exchanger.

\begin{abstract}
This study focuses on investigating the effect of protruding branching duct on laminar fluid field in $90^{\circ}$ dividing pipe junction of mini heat exchanger. The ducts under study, main and branch side, are of minichannel scale where the corresponding diameters are 3.96 and $1.6 \mathrm{~mm}$ respectively. First, numerical validations are given based on the experimental measurements of Cho et al. [1] for the case of non-protruding branching duct $(h=0, \beta \neq 0)$ and also comparing with Poiseuille flow for nonprotruding case with no flow in the branch side $(h=0, \beta=0)$ where good agreements were found in each case. Second, the degree of protrusion in pipe branching duct is characterized studying and analyzing two cases of protrusions $\left(h=D_{1} / 4\right.$ and $\left.h=D_{1} / 2\right)$. The effects of branching flow rate ratio $\beta$ on local pressure changes and the velocity profile are presented and examined in both main and branching ducts. Finally, the influence of the extent of protruded branch on velocity field and the local pressure drop are documented as expected. The results shown a strong effect of protrusion on hydrodynamic flow.
\end{abstract}

\section{INTRODUCTION}

The dividing flow in manifold systems is extensively employed in many industrial processes, engineering and biomedical applications, especially in heat exchangers devices. Flow distribution in these systems depends strongly to the ramifications designs and shapes. Protruding branching duct is one of the most relevant configuration of these because of the high change of flow field in dividing arrangement [2]. This paper is concerned with flow in piping system equipments when an extension of branching duct is welded within the main duct of heat exchanger manifold as shown in Fig. 1.

For three dimensional flow of dividing pipe junction no investigations were revealed in the literature about protrusion effect of branch side into main duct. For two dimensional distribution Tavers and Worek [2] studied numerically the effect of protruding branching duct in a planar 90 degree bifurcation. They reported that the velocity field and pressure drop were strongly influenced by the extent of the branching duct protrusion. Yang and Tsai [3] studied fluid flow and heat transfer of turbulent regime in a planar arrangement of protruding branching duct and they found that protrusion increased significantly the pressure drop in the region of the protrusion. Several investigation have been carried out on protruding branching duct for turbulent two-phase flow distribution in headers. Zughbi [4], Ren and Hrnjak [12] 
and Pei et al. [14] studied numerically and experimentally the effect of protrusion on turbulent mixing flow and they found that the two-phase distribution depends strongly on the geometry and configuration of the headers. Horiki and Osakabe [5] studied experimentally distribution behavior of water with a gasphase in a horizontal header with four vertical dividing protruded pipes. They reported that this system contributed to raise the heat transfer efficiency in the components and by increasing the protruding length the non-uniform distribution of water was suppressed. Other authors, like Kim and Han [8], Kim et al. [9], Wang J. [10], Lee and Lee [11] and Koyama et al. [13], found the same conclusions when the branch side is a rectangular duct and they revealed that the protrusion improves the headers to distribute the phases more evenly.

Ramification of non-protruding branching duct or T-junction has been previously studied by several researchers. Karino et al. [6] reported that in laminar flow of branching duct three areas of recirculation may be taken place around branch site depending on the inlet flow rate and the dividing flow rate ratio $\beta$. Cho et al. [1] carried out experimental investigation on local pressure changes and flow along the main duct examining the effect of the flow rate ratio, upstream Reynolds number, and branch angle. They found a significant influence of flow rate ratio on the pressure distribution. For two dimensional branching duct Karino and Goldsmith [7] studied the effect of the branching angle and diameter ratio on flow patterns. Also for planar rectangular dividing T-junction Liepsch et al [15] carried out measurements and numerical calculations using Navier-Stokes equations and finite-difference method. For the same Tjunction configuration Hayes et al. [16], Khodadadi [17] and Shaboury et al. [19] reported that the sizes of the two recirculation zones increase with the flow rate ratio. Yung et al. [18] and Neary and Sotiropoulos [20] investigated numerically the three-dimensionality case of T-junction with rectangular

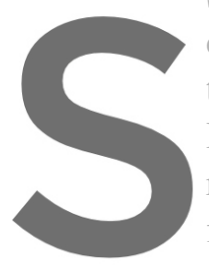
cross-section. Neary and Sotiropoulos [20] replica
that the flow was three-dimensional and flow separ
Recently Haller et al. [21] carried out experimental
rectangular cross section. He revealed that the use
fluid enhances heat transfer.
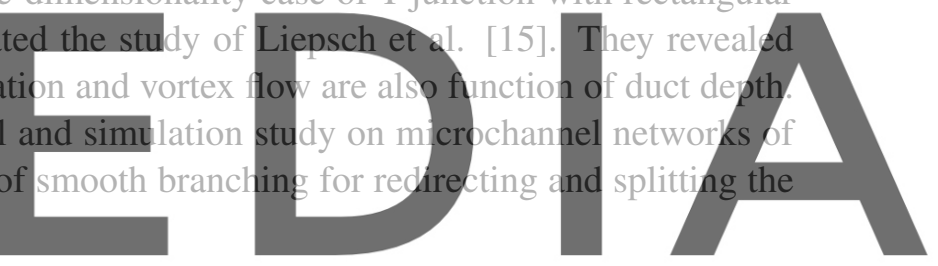

\section{Register for free at https//www.scipedia.com to download the version without the watermark}

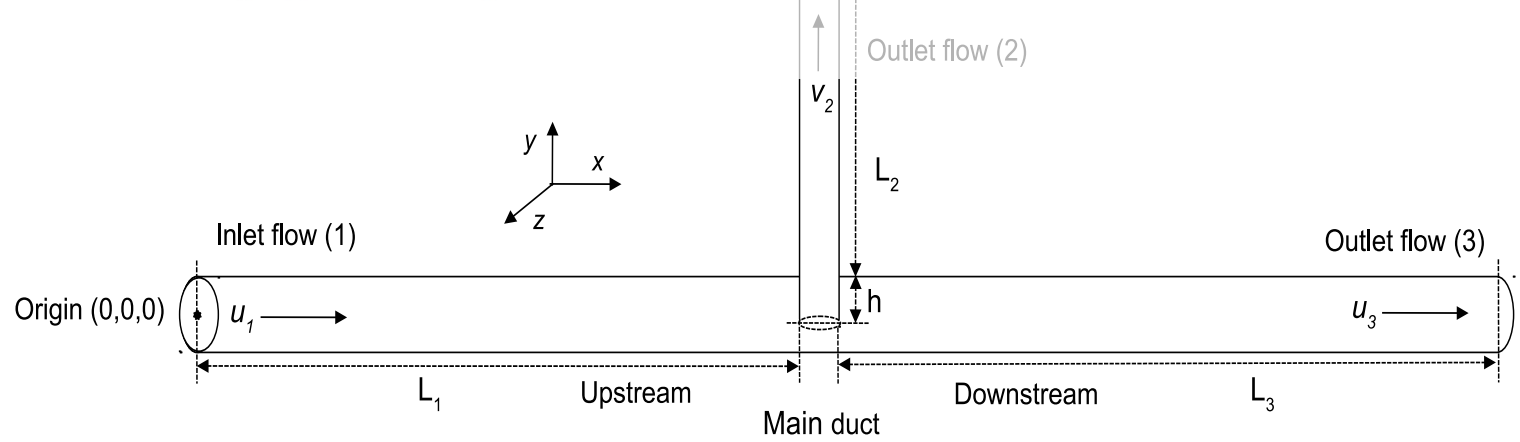

Figure 1: Geometry of protruding branching channel

In the present study computational Fluid Dynamics (CFD) investigation is performed on laminar fluid flow in $90^{\circ}$ protruding branching duct of dividing mini pipe system. The results are compared with the experimental measurements of Cho et al. [1] and the study is focused on the analysis of flow distribution 
in order to evaluate the influence of protrusion degree, the effect of flow rate ratio on pressure drop and flow circulation in main duct and protruded mini branch side.

\section{MATHEMATICAL MODEL}

The mathematical model is based on non-dimensional Navier-Stokes equations given as follows:

The equation of conservation of mass (continuity equation):

$$
\nabla \cdot U=0
$$

The equation of conservation of momentum:

$$
\frac{\partial U}{\partial t}+(U \cdot \nabla) U=-\nabla p+\frac{1}{R e} \nabla^{2} U
$$

$R e$ is the dimensionless Reynolds number defined as:

$$
R e=\rho u_{1} D_{1} / \mu
$$

Where $\rho$ and $\mu$ are the density and the dynamic viscosity of the working fluid. $D_{1}$ and $u_{1}$ are the diameter and inlet velocity of main duct, respectively.

The flow is supposed to be steady, laminar, incompressible and Newtonian with constant properties and no body forces.

The domain under study is based on the geometry of Cho et al. [1] where the main duct is of diameter

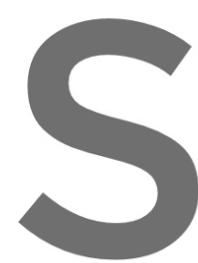
$D_{1}=D_{3}=3.96 \mathrm{~mm}$ and 1 $D_{2}=1.6 \mathrm{~mm}$ and length $L$ at the centre of main inle main duct with $h$ degree o protruding branching s incoming flow and flow at mini branch side:

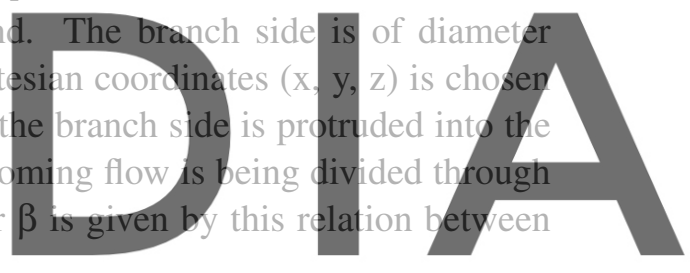

\section{Register for free at https//www.scipedia.comp toßolownload the version without the watermark}

The following boundary conditions are assumed:

- At solid boundaries we suppose no-slip velocity condition.

- At inlet of main duct fully developed velocity flow profile is fixed according to analytical solution:

$$
u=2 u_{1}\left[1-\left(\frac{r}{r_{0}}\right)^{2}\right]
$$

Where $r$ is the radial parameter and $r_{0}=D / 2$.

- At outlet of main duct a reference of zero pressure is fixed.

- At outlet of mini branch side a fully developed profile is specified.

\section{VALIDATION OF SIMULATION VS. EXPERIMENTS}

For the numerical simulation the domain is discretized with structured mesh as ilustrated in Fig. 2. For non-protruding branching duct three meshes were performed in order to validate numerical simulation: $M_{1}, M_{2}$ and $M_{3}$ of 13005,52086 and 165280 control volumes respectively. 


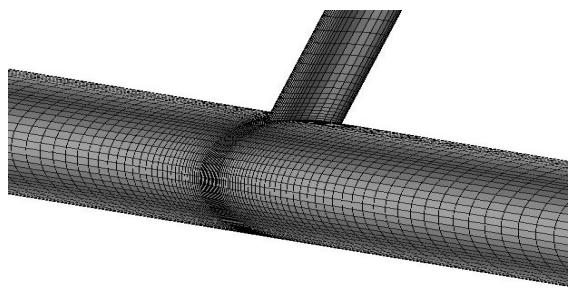

a) 3D front view of mesh

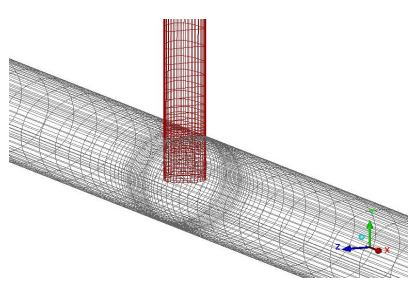

b) Wall mesh of protruding branching

Figure 2: Views of the mesh used

The results reported in this work have been performed with the TermoFluids Computational Fluid Dynamics (CFD) software package [23] which is an intrinsic 3D parallel CFD object oriented code. It is based on the fully conservative finite volume of second order schemes for spatial discretization and second order explicit time integration. The pressure velocity linkage is solved by means of an explicit fractional step procedure.

Mesh-independence tests are conducted for $33 \%$ sugar-water solution with viscosity of $\mu=4$ centipoise and density of $\rho=1142.0 \mathrm{~kg} / \mathrm{m}$ at $19^{\circ} \mathrm{C}$. Two cases of validation can be discussed:

- Case of ( $h=0, \beta=0)$ : non-protruding branching duct and zero flow in mini branch side (Fig. 3a).

Case of ( $h=0, \beta \neq 0)$ : non-protruding branching duct and flow through the branch side (Fig. 3b).
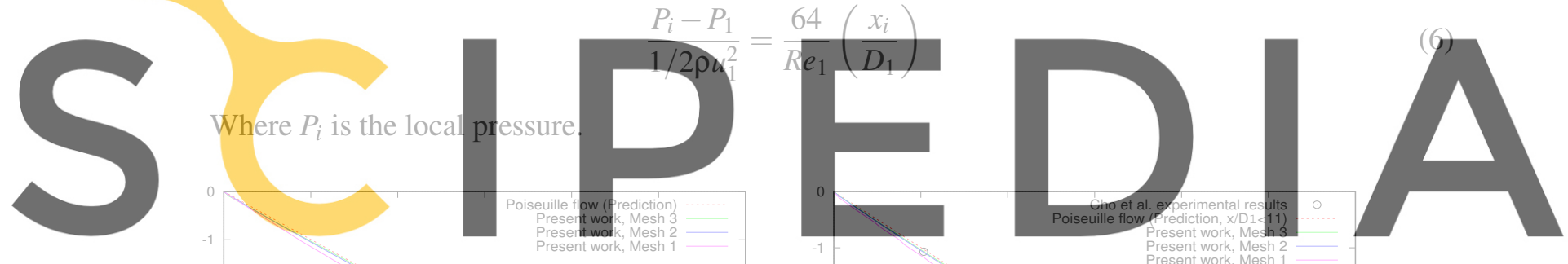

Register for free at https//www.scipedia.com

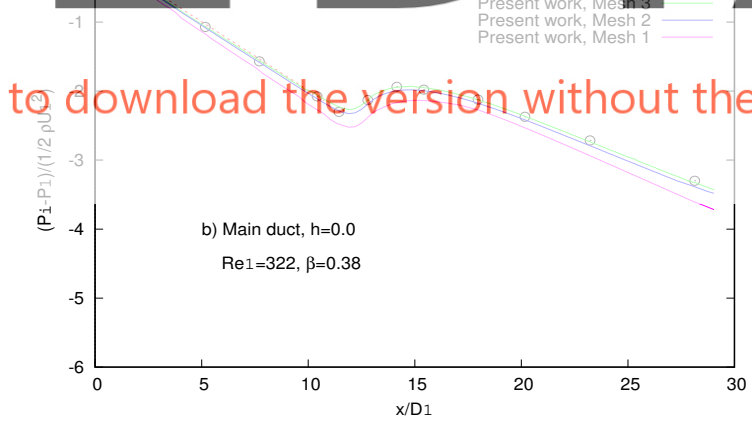

Figure 3: Comparison with Poiseuille flow $(\beta=0)$ and measurements of Cho et al. $[1](\beta \neq 0)$

The results are given in terms of dimensionless pressure change coefficient (left-hand side of equation 6) along the main duct. The local pressure $P_{i}$ is evaluated along the wall opposite the branch where measurements of Cho et al. [1] were studied.

The numerical results given in Fig. 3 indicate that the meshes $M_{2}$ and $M_{3}$ agree well with Poiseuille flow for $\beta=0$ and with experimental measurements for $\beta=0.38$. For two cases it was found that the meshes $M_{3}$ and $M_{2}$ present a mean errors around $1 \%$ and $2 \%$ respectively however the coarse mesh $M_{1}$ presents a 
mean errors between $8 \%$ and $9 \%$. Because of the small difference between meshes $M_{2}$ and $M_{3}$ the mesh $M_{2}$ can be considered sufficient for grid independent solution. Consequently basing on the mesh $M_{2}$ the mesh for the case of protruding branching was performed increasing density around the protruded mini branch side into main duct, it was of 80122 control volumes.

\section{RESULTS}

\subsection{Effect of protrusion degree on local pressure drop}

For inlet Reynolds $R e_{1}=322$ the results of the effect of protrusion degree on local pressure drop are given in Fig. 4 for the case of no flow at the branch side $(\beta=0)$ and in Fig. 5 for flow at the branch side $(\beta=0.38)$. For the main duct the results are given along the wall opposite the branch and for protruded branch side the results are given along the centerline from the wall opposite the branch $\left(y=-D_{1} / 2\right)$.
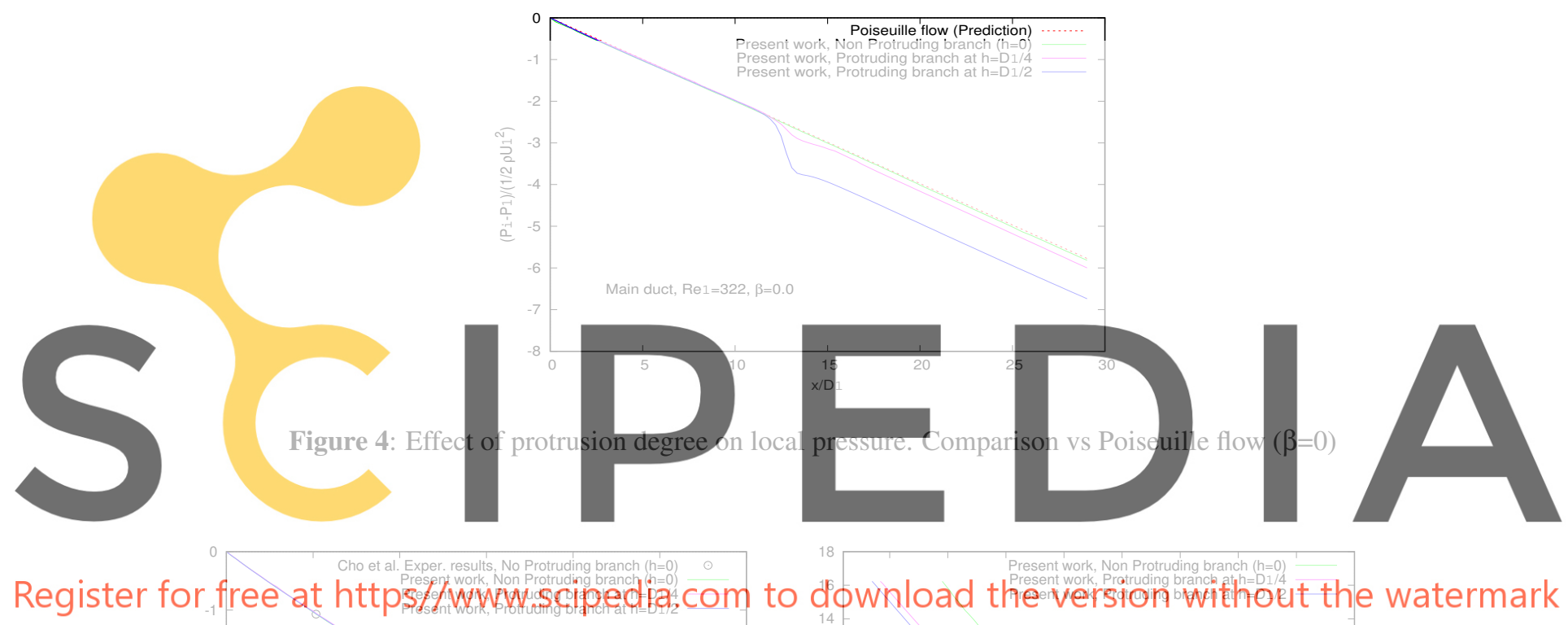

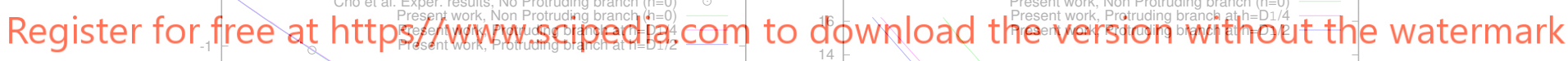
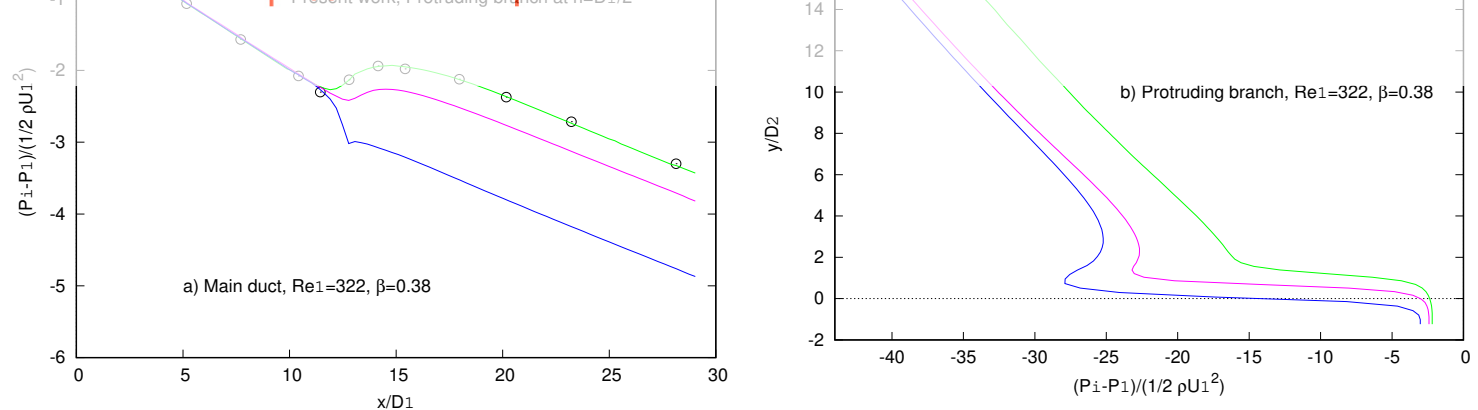

Figure 5: Effect of protrusion degree on local pressure field in main and branch ducts $(\beta=0.38)$

For the effect of protrusion degree two cases of protrusion are examined, at $h=D_{1} / 4$ and at $h=D_{1} / 2$. It was observed, for $\beta=0$ (Fig. 4) that the presence of protrusion into main duct causes:

- High decreasing of local pressure in upstream main duct close to the protruding branch location $\left(x / D_{1}=12.62\right)$ which returns to increase and recuperate the Poiseuille flow type just passing the 
protruded branch side site $\left(x / D_{1}=13.03\right)$.

- This effect increases with increasing protrusion size.

For the case of flow at branch side $(\beta=0.38)$ the results are given in Figs. $5 \mathrm{a}$ and $5 \mathrm{~b}$ for main duct and branch side respectively. These results indicate that:

- In main duct, the pressure drop increases with increasing of protrusion degree, that is the case of $h=D_{1} / 2$, presenting a significant higher pressure drop than the case of $h=D_{1} / 4$ (Fig. 5a).

- Comparing Fig. 5a with Fig. 4, for example for the case of $h=D_{1} / 2$, it is observed that after the branch location the main duct pressure drop decreases with increasing branching flow from 0 to 0.38 , reflecting the reduced mass flow at main duct outlet.

- At the entrance of mini branch side (Fig. $5 \mathrm{~b},-1 \leq \mathrm{y} / D_{2} \leq 3$ ) pressure profile changes significantly. This effect is more pronounced as protrusion increases, as also found by Tavers et al. [2] for a two dimensional case. Then the pressure evolves along the branch reaching fully developed profile type as expected.

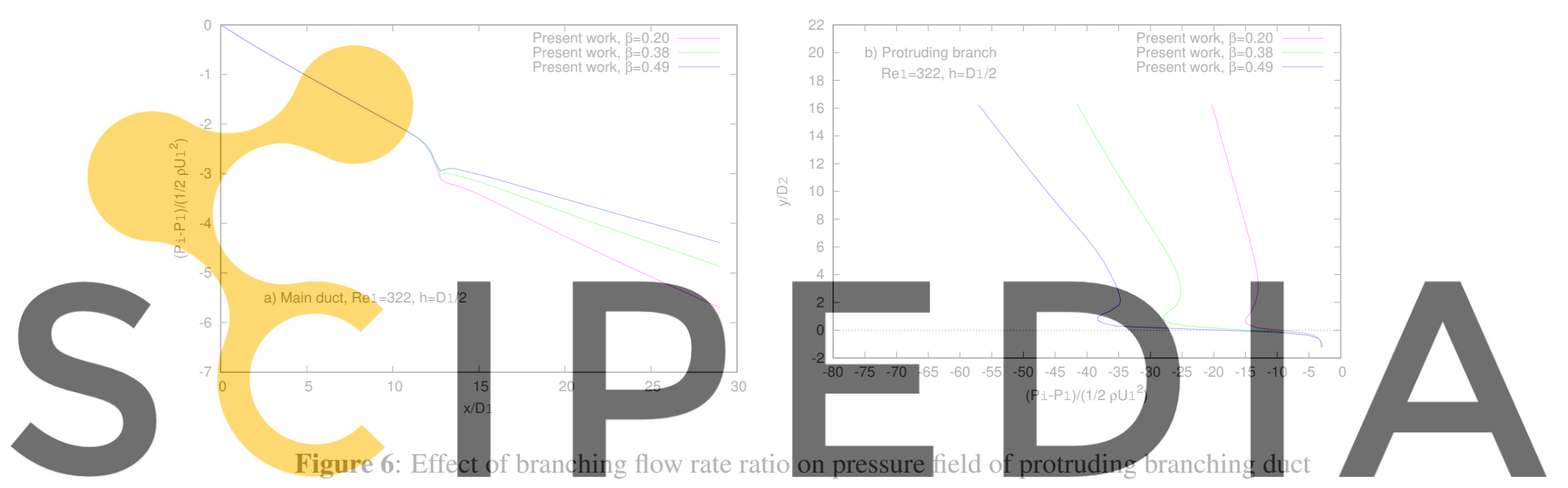

Register for free at https//www.scipedia.com to download the version without the watermark 4.2 Effect of flow rate ratio on local pressure field of protruding branching duct

The effect of dividing flow ratio on local pressure is given for the case of protruding branch side, at main centerline $h=D_{1} / 2$ and for $\beta$ from 0.2 to 0.49 . The results given in Figs. 6a and $6 \mathrm{~b}$ for inlet Reynolds number $\operatorname{Re}_{1}=322$ show that:

- For main duct the pressure drop increases with decreasing branching flow rate ratio, as observed by Cho et al. [1] in the experimental measurements for non-protruding branching case.

- For protruded mini branch side the inverse is found, that is the pressure drop increases with increasing branching flow rate ratio, because of the increase of mass flow rate.

\subsection{Velocity profile of protruding branching duct}

The study of protrusion effect on velocity profile for inlet Reynolds $R e_{1}=322$ and $h=D_{1} / 2$ is given, for branching flow rate ratio $\beta=0.0$ and 0.38 . The results are compared with Poiseuille velocity profile.

The results along main duct are presented in Fig. 7, showing that:

- Velocity profile changes around the upper corner just upstream protruding site (location $\mathrm{x}=0.0495$ ) to the reverse direction where pressure drop increases due to presence of protrusion. A small zone 

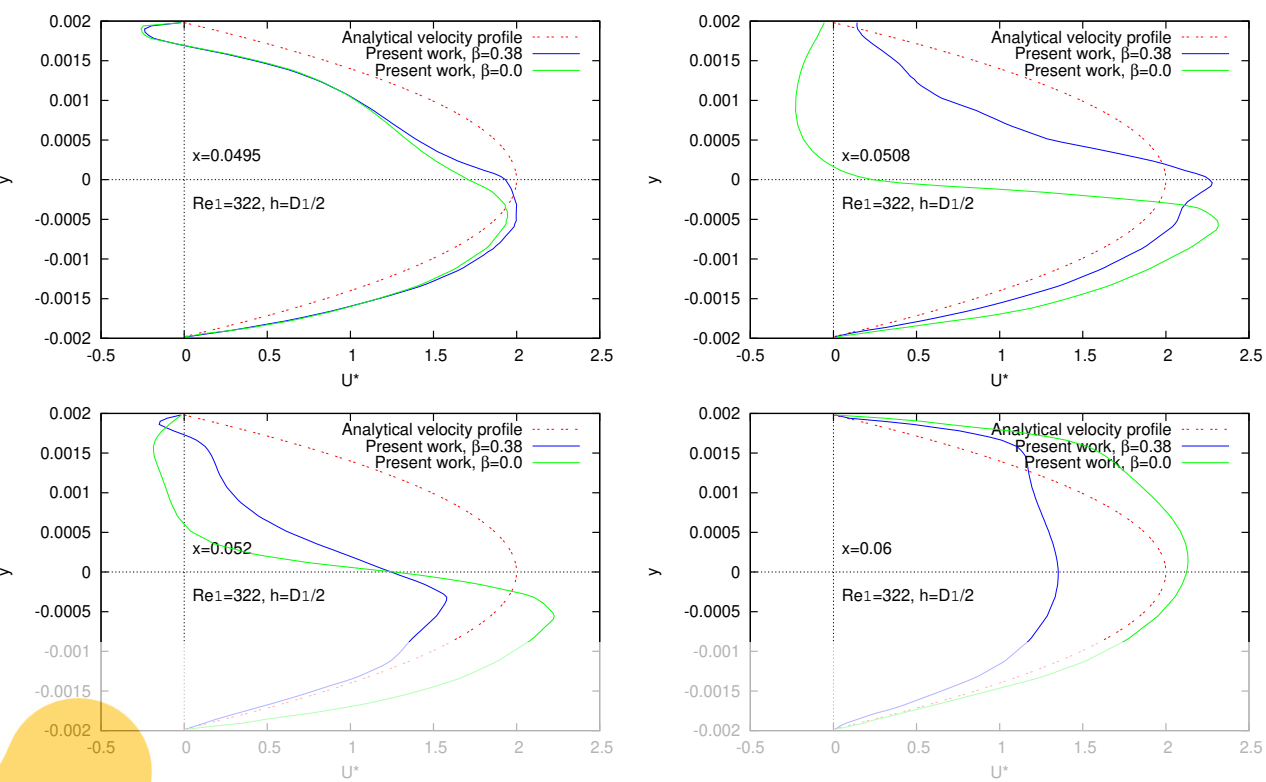

Figure 7: Velocity profile of protruding branching duct. Main duct for $\beta=0.0$ and $\beta=0.38$
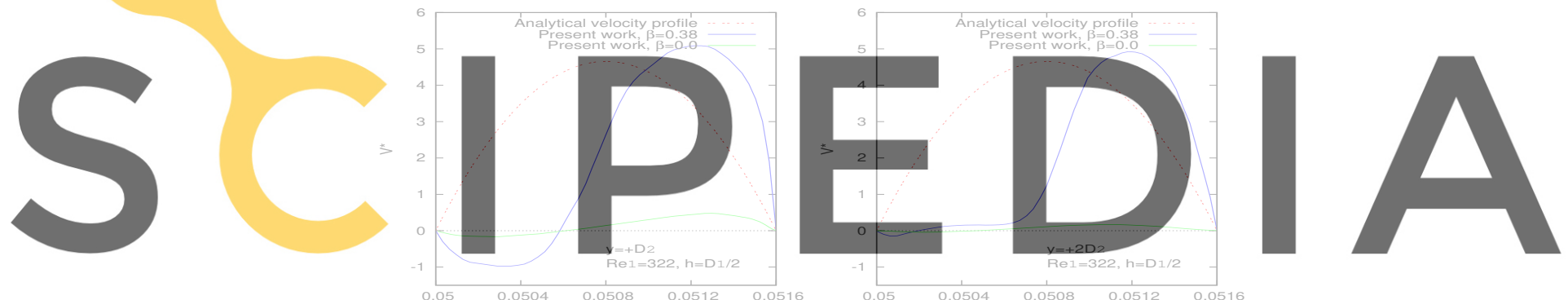

Register for free at https//www. scipe dia.com to download the version without the watermark
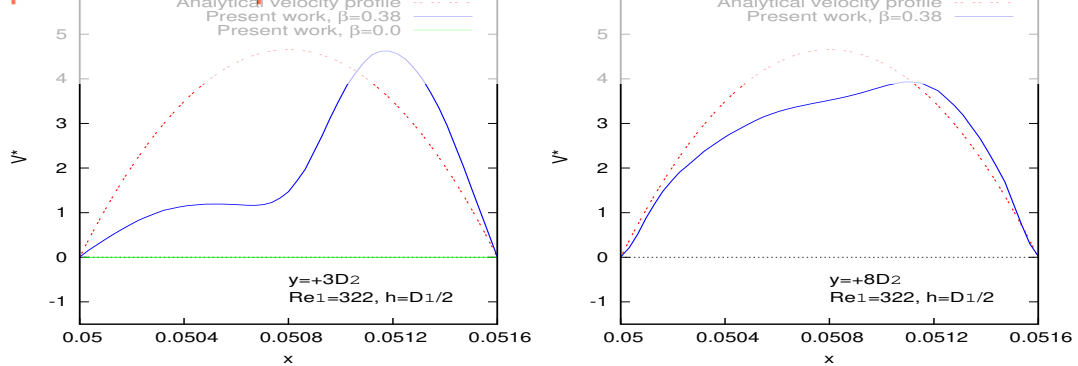

Figure 8: Velocity profile of protruding branching duct. Branch side for $\beta=0.0$ and $\beta=0.38$

of recirculation flow takes place.

- For $\beta=0.0$, at just protruded branch (location $x=0.0508$ ), streamwise velocity becomes negative, reflecting a counterclockwise recirculation in the first branch diameters (not net flow, acting as a hole). 
- A second recirculation zone was found in the upper corner just downstream protruding branching duct (location $\mathrm{x}=0.052$ ), while the velocity profiles also show the impact of the protrusion with lower velocities on the upper half of the duct. For the case of $\beta=0.38$, the maximum velocity decreases due to the effect of dividing flow rate.

- Velocity profile begins a recovery process along main duct downstream (location $\mathrm{x}=0.06,2 D_{1}$ ), with a quicker recuperation for the case of $\beta=0.0$. For $\beta=0.38$, the fully developed flow is reached approximately from $7 D_{1}$.

The results along mini branch side are given in Fig. 8, indicating that:

- At one diameter of branch inlet $\mathrm{y}=+D_{2}$, velocity profile shows a reverse flow on the left half, with a stronger recirculation zone for $\beta=0.38$.

- At the location of $y=+2 D_{2}$, the intensity of recirculation decreases significantly and then disappears at $\mathrm{y}=+3 D_{2}$. Reattachment flow takes place between these two locations.

- As seen at $\mathrm{y}=+8 D_{2}$, local velocities continue developing along mini branch side, until recuperating developed profile.

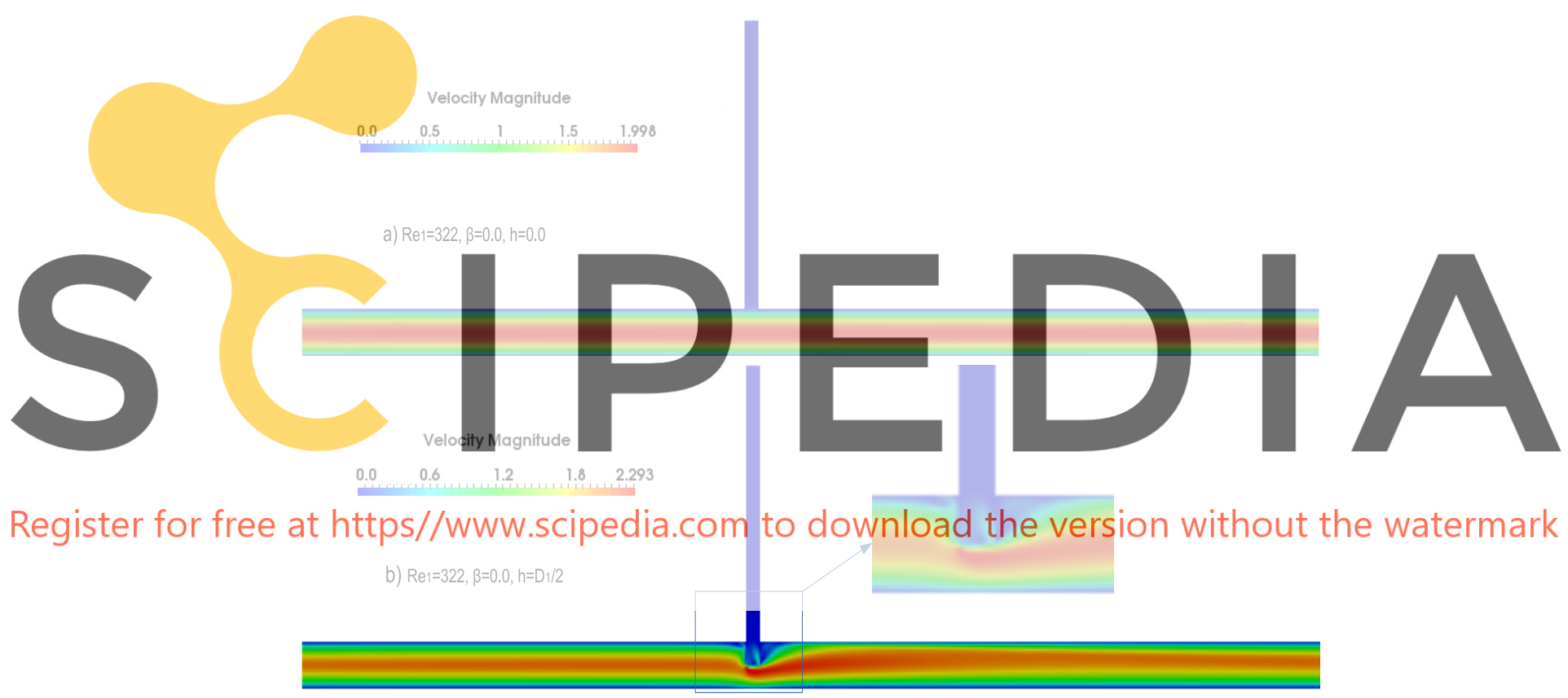

Figure 9: Maps of velocity flow along centerline. Cases of $h=0$ and $h=D_{1} / 2$ for $\beta=0.0$

\subsection{Maps of velocity profile of protruding branching duct}

Results in terms of maps of velocity profile are given in Figs. 9 and 10 for inlet Reynolds number of $R e=382$ and protrusion degree of $h=D_{1} / 2$. These are examined for both cases of $\beta=0.0$ and 0.38 .

For no flow at the branch side $(\beta=0.0)$ :

- For the case without protrusion effect $(h=0)$, Fig. 9a reflects the expected parabolic Poiseuille velocity profile.

- For $h \neq 0.0$ (Fig. 9b) protrusion effect takes place as an obstacle. Accordingly, velocity profile 


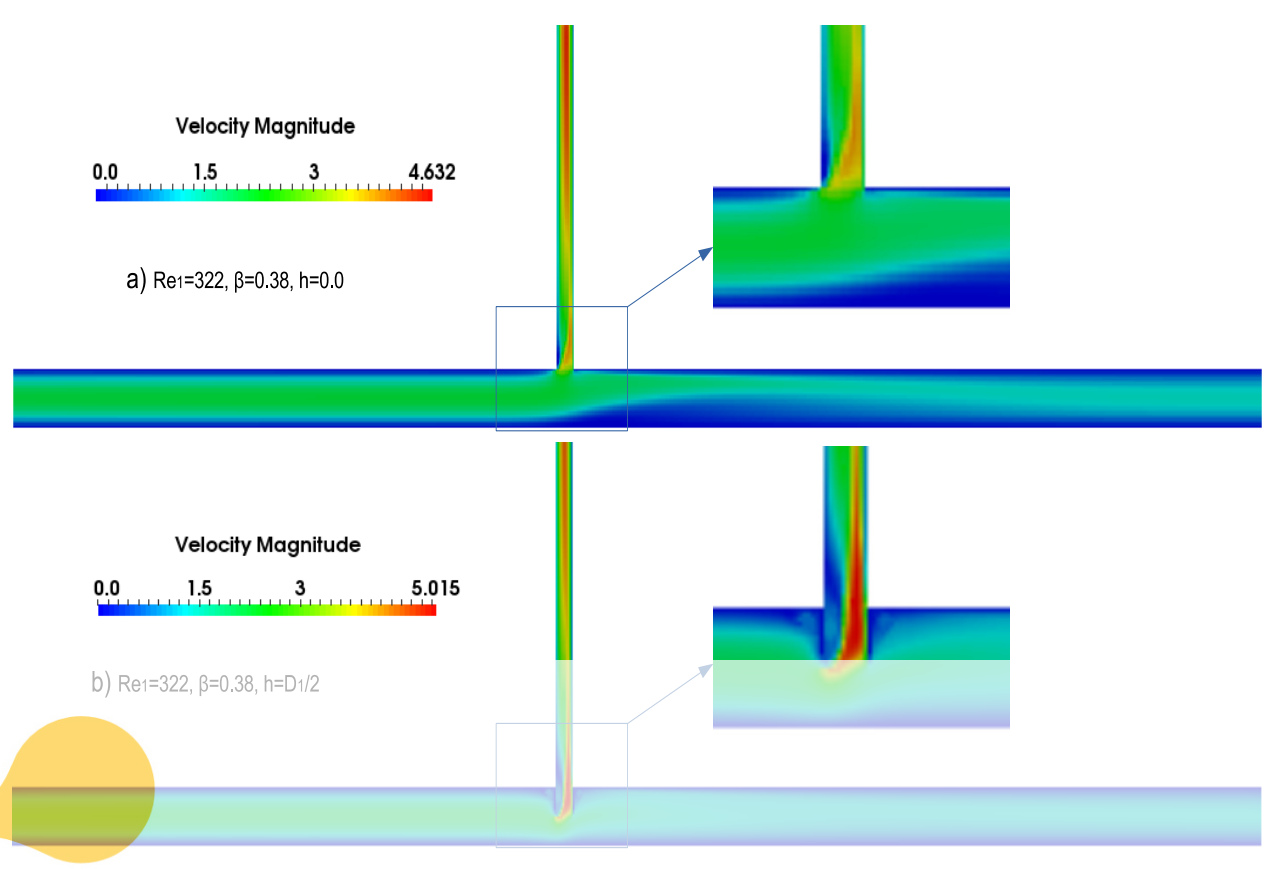

Figure 10: Maps of velocity flow along centerline. Cases of $h=0$ and $h=D_{1} / 2$ for $\beta=0.38$
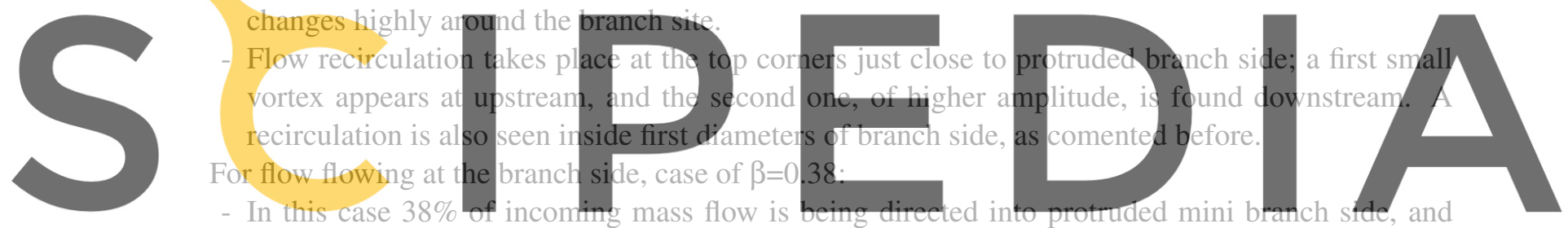

velocity flow starts developing from branch inlet.

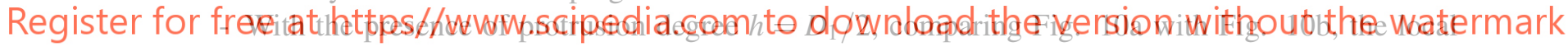

velocity increases significantly at the downstream part of protruded branch entrance, as the main duct velocity is higher in the centreline.

- As consequence of incoming mass flow part conducted into branch side (Fig. 10 a and b) local velocity decreases along main duct downstream.

- At mini branch side entrance recirculation flow appears at first diameters, the corresponding size and intensity increase with the presence of protrusion degree (Figs. 10a and 10b).

\section{CONCLUSIONS}

In this work numerical investigation of hydrodynamic effects of protruding branching duct on a dividing junction (employed in heat exchangers) was studied. The following consequences were found:

- In both ducts, main and mini protruded branch side, pressure drop increases significantly with increasing protrusion degree $(h)$.

- Pressure drop in main duct increases with decreasing branching flow rate ratio $\beta$, however in protruded mini branch side it increases with increasing $\beta$.

- In main duct local velocity decreases just main upstream close to protruded branch due to protru- 
sion effect. First recirculation and reverse flow were detected. Second flow circulation region was found at the upper part of downstream main duct close to protruded branch side.

- In mini branch side local velocity increases significantly at the entrance, on the downstream side.

- Third recirculation region was observed at entrance of mini protruded branch side and its intensity and size increase with increasing protrusion degree.

This study allows future characterization of dividing junction pressure losses. This information would enhance heat exchanger flow distribution prediction, while also opens space for its control.

\section{ACKNOWLEDGEMENTS}

Carles Oliet, as a Serra Húnter lecturer, acknowledges the Catalan Government for the support through this Programme.

\section{REFERENCES}

[1] Y. I. Cho, L. H. Back and D. W. Crawford. Experimental Investigation of Branch Flow Ratio, Angle, and Reynolds Numbers on the Pressure and Flow Fields in Arterial Branch Models. J. of Biomechanical engineering 107 (1985) 256-267.

[2] T. G. Tavers and W. M. Worek. Laminar fluid flow in a planar 90 degree bifurcation with and without a protruding branching duct. J. of Fluids engineering 118 (1996) 81-84.

[3] Yue-Tzu Yanga and Tsu-Yi Tsaia. Numerical calculation of turbulent flow in a planar bifurcation with a protruding branching duct. Numerical Heat Transfer Part A. 34 (1998) 61-74.

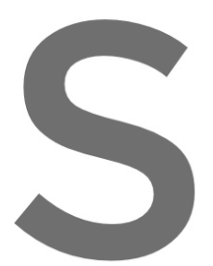

[4] H. D. Zughbi. Effects Research and Desig

[5] S. Horiki, M. Osaka of the Japan Society

[6] T. Karino, H. H. M.
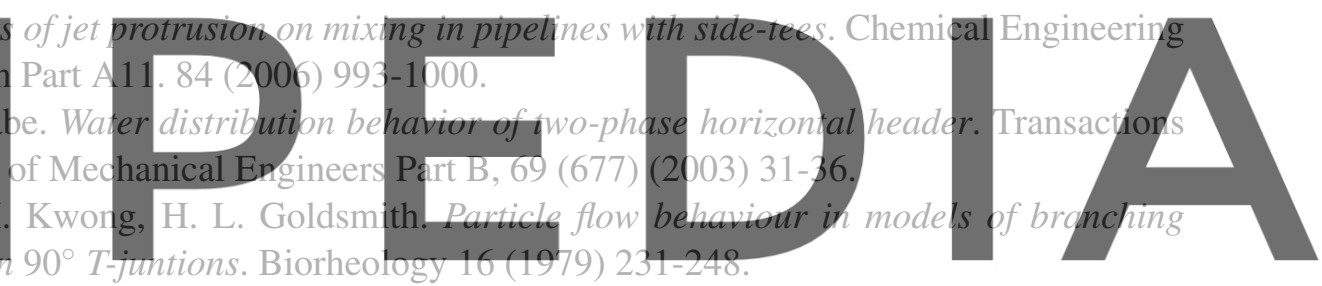

[7] T. Karino and H. L. Goldsmith. Particle flow behavior in models of branching vessels. II. Effects of

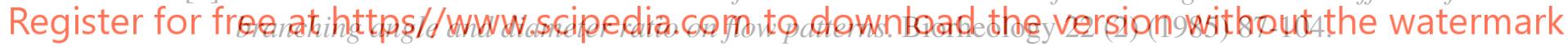

[8] Nae-Hyun Kim and Sung-Pil Han. Distribution of air-water annular flow in a header of a parallel flow heat exchanger. International Journal of Heat and Mass Transfer 51 (2008) 977-992.

[9] Nae-Hyun Kim, Eul-Jong Lee and Ho-Won Byun. Improvement of two-phase refrigerant distribution in a parallel flow minichannel heat exchanger using insertion devices. Applied Thermal Engineering 59 (2013) 116-130.

[10] Junye Wang. Design method of flow distribution in nuclear reactor systems. Chemical Engineering Research and Design 91 (2013) 595-602.

[11] Jun Kyoung Lee and Sang Yong Lee. Distribution of two-phase annular flow at header-channel junctions. Experimental Thermal and Fluid Science 28 (2004) 217-222.

[12] T. Ren, P. Hrnjak. Pressure drop in D shaped cylindrical headers of parallel flow MCHXs: Pressure loss coefficients for single phase flow. Applied Thermal Engineering 65 (2014) 111e123.

[13] Shigeru Koyama, Agung Tri Wijayanta, Ken Kuwahara and Shirou Ikuta. Developing Two-Phase Flow Distribution in Horizontal Headers With Downward Minichannel-Branches. International Refrigeration and Air Conditioning Conference. Paper 745 (2006).

[14] Kaikai Pei, Ruijiang Li and Zibin Zhu. Effects of Geometry and Process Conditions on Mixing 
Behavior of a Multijet Mixer. Industrial and Engineering Chemistry Research 53 (2014) 1070010706.

[15] D. Liepsch, S. Moravec, A. K. Rastogi and N. S. Vlachos. Measurements and calculations of laminar flow in a ninety-degree bifurcation. J. of Biomechanics 15 (7) (1982) 473-485.

[16] R. E. Hayes, K. Nandakumar and H. Nasr-El-Din. Steady laminar flow in a 90 degree planar branch. Computers and Fluids 17 (4) (1989) 537-553.

[17] Khodadadi JM, Nguyen TM, Vlachos NS. Laminar forced convective heat transfer in a twodimensional $90^{\circ}$ bifurcation. Numerical Heat Transfer 9 (1986) 677-695.

[18] Yung CN, De Witt KJ and Keith Jr TG. Three-dimensional steady on through a bifurcation. Journal of Biomechanical Engineering (ASME) 112 (2) (1990) 189-197.

[19] A. M. F. El-Shaboury, H.M. Soliman and S. J. Ormiston. Performance evaluation of branching and impacting tee junctions for laminar forced-convection applications. Int. J. of Thermal Scienes 42 (2003) 713-723.

[20] V. S. Neary and F. Sotiropoulos. Numerical investigation of laminar flows through 90-degree diversions of rectangular cross-section. Computers and Fluids 25 (2) (1996) 95-118.

[21] D. Haller, P. Woias and N. Kockmann. Simulation and experimental investigation of pressure loss and heat transfer in microchannel networks containing bends and T-junctions. Int. J. of Heat and Mass Transfer 52 (2009) 2678-2689.

[22] R. K. Shah and A. L. London. Laminar flow forced convection in ducts. Handbook of 1978 Edition.

[23] Termo Fluids S.L., Webpage: www.termofluids.com 